\title{
TAYLOR EXPANSION OF IMPLICIT FUNCTIONS DEFINED BY LINEAR EQUATIONS OF VARIABLES
}

\author{
Kazuto Asai
}

\begin{abstract}
Let $F$ be a monoid of countably many functions holomorphic at $y^{0}$, and $\left(x_{f}\right)_{f \in F}$ be a set of independent variables. We set $F_{*}=F-\{1\}, x_{*}=\left(x_{f}\right)_{f \in F_{*}}$. Let $\left(F_{1}, F_{2}, \ldots\right)$ be an increasing sequence of finite subsets of $F$ such that $\bigcup_{i \geq 1} F_{i}=F$. For $i \geq 1$, let $A_{i}$ denote the ring of all functions of $\left(x_{f}\right)_{f \in F_{i}}$, holomorphic at $\left(x_{1},\left(x_{f}\right)_{f \in F_{i}-\{1\}}\right)=$ $\left(x_{1}^{0}, 0\right)$. Define $A=\operatorname{proj} \lim A_{i}$. Consider the implicit function $y \in A$ defined by $g(y)=\sum_{f \in F} x_{f} f(y)\left(y\left(x_{1}^{0}, 0\right)=y^{0}\right)$. We have the Taylor expansion of $y$ at $x_{*}=0$ :$$
y=g^{-1}\left(x_{1}\right)+\sum_{\alpha}\left(\frac{d^{|\alpha|-1}}{d x_{1}^{|\alpha|-1}} \frac{\prod_{f \in F_{*}} f^{\alpha(f)}\left(g^{-1}\left(x_{1}\right)\right)}{g^{\prime}\left(g^{-1}\left(x_{1}\right)\right)}\right) \frac{x_{*}^{\alpha}}{\alpha !},
$$

where the sum runs over all maps $\alpha: F_{*} \rightarrow\{0,1,2, \ldots\}$ such that $|\alpha|:=\sum_{f \in F_{*}} \alpha(f)$ are positive finite.

\section{The main result}

Let $y$ be the implicit function defined by the equation: $g(y)=\sum_{i=1}^{s} x_{i} f_{i}(y)$, where $f_{1}=1$, and $g, f_{2}, \ldots, f_{s}$ are holomorphic at $y^{0}=y\left(x_{1}^{0}, 0, \ldots, 0\right)$. The purpose of the paper is to obtain the Taylor expansion of $y$ at $\left(x_{2}, \ldots, x_{s}\right)=0$. Also we apply our method to the general case: $f\left(x_{1}, \ldots, x_{n} ; y\right)=0$, with $f_{y}\left(c_{1}, \ldots, c_{n} ; y^{0}\right) \neq 0$. In this study, we lay stress on concrete calculation of the expansion coefficients. Our formula is described only by the derivatives of brief 1-variable functions and it is very useful for giving an explicit expression of the series in each case. For convenience, we deal with complex analytic functions of infinitely many variables defined as an element of the projective limit of differential rings, while the theorem could be stated by the use of ordinary analytic functions. Let $I$ be a directed set, and $\left(F_{i}\right)_{i \in I}$ index sets such that $F_{i} \subset F_{j}$ whenever $i<j$. Let $A_{i}(i \in I)$ be differential rings with derivations $\left(\delta_{f}\right)_{f \in F_{i}}$. Let $\left(A_{i}, \psi_{i j}\right)\left(\psi_{i j}: A_{j} \rightarrow A_{i}\right)$ be a projective system such that $\psi_{i j} \circ \delta_{f}=\delta_{f} \circ \psi_{i j}$ $\left(f \in F_{i}\right)$. Now we introduce the projective limit:

1991 Mathematics Subject Classification: Primary 32A05, 33B99, 39B32, Secondary 05A17.

Key words and phrases: Taylor expansion, the projective limit of differential rings, implicit functions, monoid of holomorphic functions.

Received December 15, 1999; revised August 28, 2000. 


$$
A=\operatorname{proj} \lim A_{i} \quad \text { (as a differential ring) }
$$

with derivations $\left(\delta_{f}\right)_{f \in F}\left(F=\bigcup F_{i}\right)$ that act on $y=\left(y_{i}\right)_{i \in I} \in A$ as $\delta_{f}\left(y_{i}\right):=\psi_{i j}$ 。 $\delta_{f}\left(y_{j}\right)\left(f \in F_{j}, i<j\right)$.

Let $N$ denote $\{0,1,2, \ldots\}$. Let $F$ be a monoid (with respect to ordinary product) of countably many functions holomorphic at $y^{0} \in \boldsymbol{C}$, and $x=\left(x_{f}\right)_{f \in F}$ a set of independent variables. We set $F_{*}=F-\{1\}, \quad x_{*}=\left(x_{f}\right)_{f \in F_{*}}$. Let $\left(F_{1}, F_{2}, \ldots\right)$ be an increasing (ordered by inclusion) sequence of finite subsets of $F$ such that $\bigcup_{i>1} F_{i}=F$. For $i \geq 1$, let $A_{i}$ denote the ring of all functions of $\left(x_{f}\right)_{f \in F_{i}}$ holomorphic at $\left(x_{1},\left(x_{f}\right)_{f \in F_{i}-\{1\}}\right)=\left(x_{1}^{0}, 0\right)$. Define $\psi_{i j}: A_{j} \rightarrow A_{i}$ by $\psi_{i j}(h)=\left.h\right|_{x_{f}=0}\left(f \in F_{j}-F_{i}\right)$. Let $A=\operatorname{proj} \lim A_{i}$ with derivations $\left(\partial / \partial x_{f}\right)_{f \in F}$. Let $g$ be holomorphic at $y^{0}$ such that $g\left(y^{0}\right)=x_{1}^{0}, g^{\prime}\left(y^{0}\right) \neq 0$, and consider the implicit function $y \in A$ defined by the equation:

$$
g(y)=\sum_{f \in F} x_{f} f(y) ; \quad y\left(x_{1}^{0}, 0\right)=y^{0} .
$$

For a map $\alpha: F_{*} \rightarrow N$, let $|\alpha|=\sum_{f \in F_{*}} \alpha(f), \alpha !=\prod_{f} \alpha(f)$ !, and $f^{\alpha(f)}$ denote the $\alpha(f)$ th power of $f$. Set $x_{*}^{\alpha}=\prod_{f \in F_{*}} x_{f}^{\alpha,(f)}, F_{*}^{\alpha}=\prod_{f} f^{\alpha(f)}$. (Also for a map $\alpha: F \rightarrow \boldsymbol{N}$, the above notations are defined similarly.) Let $g^{-1}$ denote the inverse function of $g$.

THEOREM 1. The Taylor expansion of $y$ at $x_{*}=0$ is given by

$$
y=g^{-1}\left(x_{1}\right)+\sum_{\substack{\alpha: F_{*} \rightarrow N \\ 0<|\alpha|<\infty}}\left(\frac{d^{|\alpha|-1}}{d x_{1}^{|\alpha|-1}} \frac{F_{*}^{\alpha}\left(g^{-1}\left(x_{1}\right)\right)}{g^{\prime}\left(g^{-1}\left(x_{1}\right)\right)}\right) \frac{x_{*}^{\alpha}}{\alpha !} .
$$

It is sometimes more convenient to consider the expansion of $y$ at $x=(0,0)$ when $g^{-1}$ is holomorphic at 0 and $g^{-1}(0)$ has simpler form than the generic $g^{-1}\left(x_{1}\right)$. Then the expansion is obtained by replacing $x_{1}$ with 0 and $x_{*}$ with $x$ in Theorem 1.

This function $y$ looks complicated due to the formulation in terms of the monoid $F$ of functions and the projective limit $A$. However, the $y$ satisfies simple partial differential equations, which are given in the next section.

\section{Partial differential equations for the $y$}

Let $y$ be as above. We prove the following lemma.

Lemma 2. If two maps $\alpha, \beta: F \rightarrow N$ satisfy that $|\alpha|=|\beta|$ and $F^{\alpha}=F^{\beta}$, then we have $\left(\partial^{|\alpha|} / \partial x^{\alpha}\right) y=\left(\partial^{|\beta|} / \partial x^{\beta}\right) y$.

Proof. We prove this by induction on $|\alpha|=|\beta|$. By concrete calculation, the lemma holds for $|\alpha|=2$. Assume the lemma is valid for $|\alpha|<m \geq 3$. Let $|\alpha|=m, \alpha(p) \neq 0$ and $\beta(q) \neq 0$. We have 


$$
\begin{aligned}
\frac{\partial^{|\alpha|}}{\partial x^{\alpha}} y & =\frac{\partial}{\partial x_{p}} \frac{\partial^{\left|\alpha^{\prime}\right|}}{\partial x^{\alpha^{\prime}}} y=\frac{\partial}{\partial x_{p}} \frac{\partial^{m-1}}{\partial x_{1}^{m-2} \partial x_{F^{\alpha^{\prime}}}} y=\frac{\partial^{m-2}}{\partial x_{1}^{m-2}} \frac{\partial^{2}}{\partial x_{p} \partial x_{F^{\alpha^{\prime}}}} y \\
& =\frac{\partial^{m-2}}{\partial x_{1}^{m-2}} \frac{\partial^{2}}{\partial x_{q} \partial x_{F^{\prime}}} y=\frac{\partial}{\partial x_{q}} \frac{\partial^{m-1}}{\partial x_{1}^{m-2} \partial x_{F^{\beta^{\prime}}}} y=\frac{\partial}{\partial x_{q}} \frac{\partial^{\left|\beta^{\prime}\right|}}{\partial x^{\beta^{\prime}}} y=\frac{\partial^{|\beta|}}{\partial x^{\beta}} y,
\end{aligned}
$$

where $\alpha^{\prime}(f)=\alpha(f)-\delta_{f, p}, \beta^{\prime}(f)=\beta(f)-\delta_{f, q} . \quad(\delta$ is Kronecker delta. $)$

By virtue of Lemma 2, we can compute the coefficients of the expansion of $y$ by $\left(\partial^{|\alpha|} y / \partial x_{*}^{\alpha}\right)\left(x_{1}, 0\right)=\left(d^{|\alpha|-1} / d x_{1}^{|\alpha|-1}\right)\left(\partial y / \partial x_{F_{*}^{\alpha}}\right)\left(x_{1}, 0\right)$, and immediately deduce Theorem 1 .

\section{Examples}

Example I. Let $F=\left\{y^{i} ; i \in \boldsymbol{N}\right\}, \quad x_{y^{i}}=x_{i}, \quad$ and $g(y)=y^{n}$; that is, $y^{n}=\sum_{i \in N} x_{i} y^{i}$. We can give another proof of the formula below first proved by $\mathrm{Hj}$. Mellin in [5]. Set $|m|=\sum m_{i}$ and $p(m)=\sum i m_{i}$.

$$
y=\frac{1}{n} \sum_{\substack{m_{1}, m_{2}, \ldots \geq 0 \\|m|<\infty}} \frac{\Gamma((p(m)+1) / n)}{\Gamma((p(m)+1) / n-|m|+1)} \cdot x_{0}^{(p(m)-n|m|+1) / n} \frac{x_{1}^{m_{1}} x_{2}^{m_{2}} \cdots}{m_{1} ! m_{2} ! \cdots} .
$$

Example II. Let $F=\left\{\exp \left(\sum_{1 \leq i \leq r} a_{i} y^{i}\right) ; a \in \boldsymbol{Z}^{r}\right\}, x_{\exp \left(a_{1} y+\cdots+a_{r} y^{r}\right)}=x_{a}, x=$ $\left(x_{a}\right)_{a \in \boldsymbol{Z}^{r}}$, and $g(y)=y$. Then $y=\sum_{a \in \boldsymbol{Z}^{r}} x_{a} \exp \left(a_{1} y+\cdots+a_{r} y^{r}\right)$. We have

$$
y=\sum_{\substack{\alpha: Z^{r} \rightarrow N \\ 0<|\alpha|<\infty}} \sum_{\substack{\lambda: \text { partitions of }|\alpha|-1 \\ \lambda_{1} \leq r}} \frac{(|\alpha|-1) !}{m(\lambda) !}\left(\prod_{i=1}^{l(\lambda)} \sum_{a \in \boldsymbol{Z}^{r}} \alpha(a) a_{\lambda_{i}}\right) \frac{x^{\alpha}}{\alpha !},
$$

where $m(\lambda)$ denotes $\left(m_{1}, m_{2}, \ldots\right)$ defined by $m_{i}=\sharp\left\{j ; \lambda_{j}=i\right\}, l(\lambda)$ denotes the length $l$ of $\lambda=\left(\lambda_{1}, \ldots, \lambda_{l}\right)$, and $m(\lambda)$ !, $\lambda$ ! denote the products of factorials of the components.

Example I is easily certified by computing the coefficients $c_{m}$ of $x^{m} / m$ ! by $c_{m}=(1 / n)\left(d / d x_{0}\right)^{|m|-1} x_{0}^{(p(m)-n+1) / n}$. Example II is shown as follows. To obtain the coefficient of $x^{\alpha} / \alpha$ ! , it suffices to calculate the $(|\alpha|-1)$ th derivative of $e^{b_{1} x_{0}+\cdots+b_{r} x_{0}^{r}}$ at $x_{0}=0$, where $b_{k}=\sum_{a \in Z^{r}} \alpha(a) a_{k}$. Each term of the derivative is characterized by the partition $\lambda$ with $\lambda_{1} \leq r$ as follows: $\lambda \rightarrow h^{\left(\lambda_{1}\right)} h^{\left(\lambda_{2}\right)} \cdots h^{\left(\lambda_{l}\right)} e^{h}$ $\left(h=a_{1} x_{0}+\cdots+a_{r} x_{0}^{r}\right)$. Thus we have the expansion.

\section{Application to the general case}

Let $f\left(x_{1}, \ldots, x_{n} ; y\right)$ be a function holomorphic at $x_{*}=\left(x_{1}, \ldots, x_{n}\right)=c, y=$ $y^{0}$, such that $f\left(c ; y^{0}\right)=0$ and $f_{y}\left(c ; y^{0}\right) \neq 0$. Let us consider the general problem to give the Taylor series of the function $y$ defined by $f\left(x_{1}, \ldots, x_{n} ; y\right)=0, y(c)=$ $y^{0}$. We make use of the expansion of $f\left(x_{1}, \ldots, x_{n} ; y\right)$ at $x_{*}=c$ to reduce the 
problem to Theorem 1. First of all, we add a dummy variable $x_{0}$, and set $x_{0}+$ $f\left(x_{1}, \ldots, x_{n} ; y\right)=0$. Then it suffices to have the expansion of $y$ at $x_{*}=c$ and put $x_{0}=0$. We obtain that for some neighborhood $\left\|x_{*}-c\right\|<\rho,\left\|y-y^{0}\right\|<\sigma$, $x_{0}+\sum_{\alpha \in N^{n}}\left(\partial^{|\alpha|} f / \partial x^{\alpha}\right)(c ; y)\left(x_{*}-c\right)^{\alpha} / \alpha !=0$, or with the notation $f_{\alpha}(y)=(1 / \alpha !)$. $\left(\partial^{|\alpha|} f / \partial x^{\alpha}\right)(c ; y)$

$$
-f_{0}(y)=x_{0}+\sum_{\alpha \neq 0} f_{\alpha}(y)\left(x_{*}-c\right)^{\alpha} .
$$

Let $F=\left\langle f_{\alpha}\right\rangle_{\alpha \neq 0}$ be the monoid generated by the functions $\left\{f_{\alpha}\right\} \quad\left(\alpha \in \boldsymbol{N}^{n}\right.$, $\alpha \neq 0)$. $\quad F$ is not finitely generated, but contains only countably many functions. Set $F_{i}=\left\langle f_{\alpha}\right\rangle_{0<|\alpha| \leq i}$ and we have $\bigcup_{i=1}^{\infty} F_{i}=F$. Let $F_{*}=F-\{1\}$. Let $A_{i}$ denote the ring of all functions of independent variables $\left(\tilde{x}_{\alpha}\right)_{|\alpha| \leq i}$ holomorphic at 0 . This formulation enables us to introduce the projective limit $A=\operatorname{proj} \lim A_{i}$ as in $\S 1$. Let $\tilde{y}$ be the element of $A$ defined by the deformed equation:

$$
-f_{0}(\tilde{y})=\tilde{x}_{0}+\sum_{\alpha \neq 0} f_{\alpha}(\tilde{y}) \tilde{x}_{\alpha} .
$$

We now expand $\tilde{y}$ by Theorem 1 , and substituting $\left(x_{*}-c\right)^{\alpha}$ for $\tilde{x}_{\alpha}$, we obtain the following expansion of $y$. To state the theorem, we use several notations: $\boldsymbol{N}^{n+}=\boldsymbol{N}^{n}-\{0\}, \quad \xi=a \operatorname{map}_{\xi} \boldsymbol{N}^{n+} \rightarrow \boldsymbol{N}, \quad|\xi|=\sum_{\alpha \in \boldsymbol{N}^{n+}} \xi(\alpha) \alpha, \quad l(\xi)=\sum_{\alpha} \xi(\alpha)$, $\xi !=\prod_{\alpha} \xi(\alpha) !$, and $F_{*}^{\xi}=\prod_{\alpha} f_{\alpha}^{\xi(\alpha)}$.

THEOREM 3. The function $y$ defined by (4.1) has the expansion:

$$
y=f_{0}^{-1}\left(-x_{0}\right)+\sum_{|\alpha|>0}\left(\sum_{|\xi|=\alpha} \frac{1}{\xi !} \frac{d^{l(\xi)-1}}{d x_{0}^{l(\xi)-1}} \frac{F_{*}^{\xi}\left(f_{0}^{-1}\left(-x_{0}\right)\right)}{-f_{0}^{\prime}\left(f_{0}^{-1}\left(-x_{0}\right)\right)}\right)\left(x_{*}-c\right)^{\alpha} .
$$

Example III. Let $p_{1}, \ldots, p_{n}, s$ be arbitrary complex numbers and set $q=$ $p_{1}+\cdots+p_{n}-e^{s}$. Let $y$ be the function defined by the following equality.

$$
p_{1} y^{x_{1}}+\cdots+p_{n} y^{x_{n}}=y+q .
$$

Then we have the series expression of $y$ below.

$$
y=e^{s}+\sum_{|\alpha|>0} x_{*}^{\alpha} \sum_{\lambda} \frac{p^{l(\lambda)} e^{-s|l(\lambda)|+s}}{m(\lambda) ! \lambda !} \sum_{i=0}^{|l(\lambda)|-1} \frac{|\alpha| ! s^{|\alpha|-i}}{(|\alpha|-i) !} \sum_{\mu} \frac{(-1)^{|\mu|-i}|\mu| !}{m(\mu) ! \mu_{1} \cdots \mu_{i}} .
$$

Here, $\lambda$ runs over all $n$-tuples $\left(\lambda^{1}, \ldots, \lambda^{n}\right)$ of partitions such that $\left|\lambda^{i}\right|:=\lambda_{1}^{i}+$ $\lambda_{2}^{i}+\cdots=\alpha_{i}$, and $\mu$ runs over all partitions with the conditions: $|\mu|=|l(\lambda)|-1$ $\left(|l(\lambda)|=l\left(\lambda^{1}\right)+\cdots+l\left(\lambda^{n}\right)\right), l(\mu)=i$. Further, we used the following notations: $p^{l(\lambda)}=p_{1}^{l\left(\lambda^{1}\right)} \cdots p_{n}^{l\left(\lambda^{n}\right)}, \lambda !=\lambda^{1} ! \cdots \lambda^{n} !, m(\lambda) !=m\left(\lambda^{1}\right) ! \cdots m\left(\lambda^{n}\right) !$

The result (4.5) is based on Theorem 3 and an expression of $(d / d u)^{n}(\log u)^{m}$. Indeed, by Theorem 3, it is shown that 


$$
y=e^{s}+\sum_{|\alpha|>0} x_{*}^{\alpha} \sum_{\lambda} \frac{p^{l(\lambda)}}{m(\lambda) ! \lambda !}\left[\frac{d^{|l(\lambda)|-1}}{d x_{0}^{|l(\lambda)|-1}}\left(\log \left(x_{0}+e^{s}\right)\right)^{|\alpha|}\right]_{x_{0}=0} .
$$

On the other hand, we have $(d / d u)^{n}(\log u)^{m}=\left(1 / u^{n}\right) \sum_{i=0}^{\min \{m, n\}}\left(m !(\log u)^{m-i}\right.$ $/(m-i) !) \sum_{\mu}\left((-1)^{n-i} n ! / m(\mu) ! \mu_{1} \cdots \mu_{i}\right)$. Now put $u=e^{s}$. Two equalities verify (4.5) as desired.

\section{REFERENCES}

[1] P. Appell, Sur les fonctions hypergéométriques de plusieurs variables, Mémor. Sci. Math., Gauthier-Villars, 1925.

[2] L. Bieberbach, Analytische Fortsetzung, Springer, 1955.

[3] P. Dienes, The Taylor Series, Clarendon Press, 1931.

[4] E. LandaU, Darstellung und Begründung einiger neuerer Ergebnisse der Funktionentheorie, Springer, 1929.

[5] Ho. Mellin, Résolution de l'équation algébrique générale à l'aide de la fonction $\Gamma, \mathrm{C} . \mathrm{R}$. Acad. Sci. Paris, 172 (1921), 658-661.

[6] M. Noumi, Expansion of the solutions of a Gauss-Manin system at a point of infinity, Tokyo J. Math., 7 (1984), 1-60.

[ 7] H. Umemura, Resolution of algebraic equations by theta constants, Tata Lectures on Theta II, Progress in Math. 43, Birkhäuser, 1984, 261-272.

Center for Mathematical SCIEnCES

UNIVERSITY OF AIZU

Aizu-Wakamatsu, Fukushima, 965-8580

JAPAN

e-mail: k-asai@u-aizu.ac.jp 